\title{
Méthodes de routine pour le dosage de la matière grasse et des protéines du lait de chèvre
}

\author{
par \\ R. GRAPPIN et R. JEUNET \\ I.N.R.A., Station Expérimentale Laitière - 39800 Poligny (1)
}

La France compte environ 150000 élevages laitiers caprins totalisant près de 830000 chèvres. En 1977, 55000 chèvres étaient soumises à un contrôle laitier régulier et il est probable que le lait livré par les producteurs aux entreprises laitières (environ 20000 producteurs) sera prochainement payé en fonction de sa composition en matière grasse et en protéines.

L'ensemble des contrôles qui seront effectués pour le contrôle laitier individuel et le paiement du lait représentera un nombre important d'échantillons de lait, qui devront être analysés par les laboratoires français.

Cette étude a pour but d'évaluer la précision et de définir les conditions d'utilisation des méthodes et appareils (méthode Noir Amido, appareils Milko-Tester, IRMA et Milko-Scan 300) déjà couramment employés pour l'analyse des laits de vache et qui seront utilisés pour les laits de chèvre.

\section{MATERIELS ET METHODES}

\section{I.1. Laits}

Les quatre-vingt-un échantillons individuels de lait de chèvre analysés dans cette étude proviennent de trois élevages de races Saanen et Alpine situés dans la région de Poligny (Jura). Les prélèvements ont été effectués au mois de mai, sur des animaux en milieu de lactation. Parallèlement, cinquante et un échantillons de lait de vache prélevés dans des bidons à l'arrivée à la laiterie ont également été analysés. Tous les échantillons proviennent du lait d'une seule traite.

(1) Cette étude fait partie d'un programme plus complet financé par un contrat D.G.R.S.T. $\mathrm{n}^{\circ} 7770564$. 


\section{I.2. Méthodes d'analyses}

\section{I.2.1. MÉTHOdeS DE RÉFÉRENCE}

I.2.1.1. Matière grasse : méthode Gerber : les résultats sont exprimés en grammes de matière grasse par $\mathrm{kg}$ de lait.

I.2.1.2. Protéines et fractions azotées : ces dosages ont été effectués sur la totalité des échantillons de lait de chèvre et seulement sur le lait de mélange des échantillons de lait de vache.

- Azote total, azote soluble (ou azote non caséine) et azote non protéique (NPN) : méthode décrite par Rowland (1938). Le dosage de l'azote est fait par la méthode Kjeldahl.

- Azote du lactosérum : dosage par la méthode Kjeldahl de l'azote du lactosérum (filtrat) obtenu après coagulation du lait par la présure commerciale et filtration sur papier.

Pour exprimer les résultats en grammes de matière azotée $(\mathrm{N} \times$ 6,39) par $\mathrm{kg}$ de lait, il est tenu compte du volume du précipité lorsque les dosages d'azote sont faits sur des filtrats (NPN, N soluble, $\mathrm{N}$ lactosérum). On utilise un coefficient commun de 0,987 pour le NPN, de 0,9935 pour l'azote soluble et le coefficient $\mathrm{K}=\frac{900-0,9 \mathrm{G}-1,45 \mathrm{M} . \mathrm{A} \text {. }}{900-0,9 \mathrm{~g}-1,45 \mathrm{~m} . \mathrm{a} \text {. }}$ pour le lactosérum dans lequel G et M.A. sont les teneurs en graisse et en MAT du lait, g et m.a. les teneurs en graisse et en MAT du sérum.

Après dosage des différentes fractions azotées, on peut calculer les composants suivants :

\footnotetext{
- taux de matière azotée totale (T. MAT)

- taux de protéines (TP)

$=\mathrm{N}$ total $\times 6,39$

- taux de caséine (TC)

- taux de protéines solubles (T.PS)

$=(\mathrm{N}$ total $-\mathrm{NPN}) \times 6,39$

- taux de protéines coagulables (T.PC)

$=(\mathrm{N}$ total $-\mathrm{N}$ soluble $) \times 6,39$

$=(\mathrm{N}$ soluble $-\mathrm{NPN}) \times 6,39$

$=(\mathrm{N}$ total $-\mathrm{N}$ sérum $) \times 6,39$

- taux de protéines non coagulables $($ T.PNC $)=(\mathrm{N}$ sérum $-\mathrm{NPN}) \times 6,39$
}

\section{I.2.2. MÉTHODES RAPIDES}

1.2.2.1. Matière grasse : appareils Milko-Tester (MT), Infra-RedMilk Analyser MK II (IRMA) et Milk-Scan 300 (MS).

1.2.2.2. Protéines du lait et du lactosérum: méthode Noir Amido (NF / V 04-216) et appareils IRMA (lait uniquement) et MS.

Tous les échantillons de lait de chèvre et tous les échantillons de lait de vache ont été analysés simultanément sur chaque appareil en utilisant le même calibrage pour les deux types de lait. Le T.PC est obtenu par différence entre le résultat obtenu sur lait et le résultat sur sérum, les appareils étant calibrés en TP pour les analyses de lait et en T.PNC pour les analyses de sérum. Ce dernier 


\section{TABLEAU 1}

Teiıeur moyenne des principaux éléments des échantillons de lait de chèvre et de lait de vache

\begin{tabular}{|c|c|c|c|c|}
\hline \multirow{2}{*}{ Composant } & \multicolumn{2}{|c|}{ Chèvre } & \multicolumn{2}{|c|}{ Vache } \\
\hline & $\bar{x}$ & $\sigma$ & $\bar{x}$ & $\sigma$ \\
\hline Matière grasse (g/kg) & 30,1 & 5,41 & 38,1 & 3,04 \\
\hline MAT (g/kg) & 28,3 & 2,27 & 32,0 & - \\
\hline $\mathrm{TP}(\mathrm{g} / \mathrm{kg})$ & 25,6 & 2,25 & 30,5 & 1,51 \\
\hline Lactose $(\mathrm{g} / \mathrm{kg})$ & 46,1 & 2,14 & 50,2 & 1,41 \\
\hline Nombre de cellules $/ \mathrm{ml}(\times 1000)$ & 1300 & - & - & - \\
\hline
\end{tabular}

TABLEAU 2

Proportion des différentes fractions azotées du lait de chèvre et du lait de vache

\begin{tabular}{|c|c|c|c|c|c|c|c|c|}
\hline \multirow{2}{*}{\multicolumn{2}{|c|}{ Lait }} & \multirow{2}{*}{$\mathrm{n}$} & \multicolumn{4}{|c|}{ p. 100 par rapport à la MAT } & \multicolumn{2}{|c|}{$\begin{array}{c}\text { p. } 100 \\
\text { par rapport } \\
\text { au TP }\end{array}$} \\
\hline & & & caséine & PS & NPN & MAC & PS & MAC \\
\hline \multirow{2}{*}{ Etude 78} & chèvre & 81 & 75,3 & 15,4 & 9,3 & 70,7 & 16,9 & 78,1 \\
\hline & vache & 49 & 78,5 & 16,7 & 4,8 & - & 17,5 & - \\
\hline \multirow{2}{*}{ Etude 72} & chèvre & 79 & 75,8 & 14,2 & 10,0 & - & 15,8 & - \\
\hline & vache & 94 & 78,1 & 16,3 & 5,6 & - & 17,3 & - \\
\hline
\end{tabular}

PS : protéines solubles.

NPN : matières azotées non protéiques.

MAC : matières azotées coagulables. 
taux représente en fait le T.PS auquel s'ajoute le caseinomacropeptide résultant de l'hydrolyse par la présure.

\section{I.3. Analyses statistiques}

Pour estimer la précision et le calibrage des différentes méthodes rapides d'analyse, nous utiliserons les définitions suivantes (Grappin, 1976) :

\section{I.3.1. PRÉcision D'Estimation}

Elle est donnée par les limites $\mathrm{T} 1$ et $\mathrm{T} 2$ de l'intervalle statistique de dispersion, limites à l'intérieur desquelles se situe, avec une probabilité de 95 p. 100, la vraie valeur yo donnée par la méthode de référence pour une valeur donnée xo de la méthode de routine à tester. Si $\sigma_{y, x}$ est l'écart-type résiduel déduit de la régression de y (méthode de référence) sur $\mathrm{x}$ (méthode de routine), la précision d'estimation de la grandeur à mesurer (T.MG ou TP) par la méthode de routine est égale à $\pm 1,96 \sigma_{y, x}$.

\section{I.3.2. CAlibrage}

La droite observée de calibrage est définie par l'équation de la droite de régression $\mathrm{y}=\mathrm{bx}+\mathrm{a}$, calculée à partir des résultats obtenus avec un grand nombre d'échantillons analysés par la méthode de référence (y) et par la méthode de routine $(\mathrm{x})$.

Nous n'avons pas évalué la répétabilité des différentes méthodes car elle ne présente pour le lait de chèvre aucune particularité qui la différencie de celle du lait de vache.

\section{RESULTATS}

\section{II.1. Composition des laits}

Nous donnons dans le tableau 1 la teneur des principaux constituants des laits de chèvre et des laits de vache qui ont été analysés dans cette étude.

Le tableau 2 groupe les résultats des dosages des fractions azotées auxquels nous avons joint les résultats d'une étude semblable faite en 1972 et portant sur soixante-dix-neuf échantillons individuels de lait de chèvre et quatre-vingt-quatorze échantillons individuels de laits de vache.

\section{II.2. Indice de réfraction de la matière grasse}

Le tableau 3 indique, pour chaque appareil, la relation entre l'indice de réfraction de la matière grasse et la différence relative entre les résultats obtenus et la méthode Gerber. 


\section{TABLEAU 3}

Relation entre l'indice de réfraction de la matière grasse $(\mathrm{x})$ exprimé en 10-4 unités de $n_{D}$ et la différence relative entre les résultats donnés par chaque appareil et la méthode Gerber (y) exprimée par : (appareil-Gerber) $\times 100 /$ Gerber.

\begin{tabular}{|c|c|c|c|c|}
\hline & & $e(n=20)$ & & $(n=26)^{*}$ \\
\hline & $r^{2}$ & $\mathrm{~b}_{y, \mathrm{x}}$ & $\mathrm{r}^{2}$ & $\mathrm{~b}_{y, \mathrm{x}}$ \\
\hline MT & 0,86 & $+0,31 \pm 0,06$ & 0,83 & $+0,15 \pm 0,03$ \\
\hline MS & 0,34 & $-0,15 \pm 0,09$ & 0,85 & $-0,20 \pm 0,03$ \\
\hline IRMA & 0,58 & $-0,31 \pm 0,12$ & 0,73 & $-0,25 \pm 0,06$ \\
\hline $\begin{array}{l}\mathrm{r}^{2}: \mathrm{c} \\
\mathrm{b}: \mathrm{c} \\
*: \mathrm{G}\end{array}$ & $\begin{array}{l}\text { coef } \\
\text { ent de } \\
\text { et } \mathrm{Je}\end{array}$ & $\begin{array}{l}\text { corrélation e } \\
\text { on de y sur } x \text {. } \\
\text { 78). }\end{array}$ & & \\
\hline
\end{tabular}

\section{TABLEAU 4}

Précisions d'estimation ( $\left.\pm 1,96 \sigma_{y, x}\right)$ exprimées en $\mathrm{g} / \mathrm{kg}$ des teneurs obtenues avec les différentes méthodes de routine utilisées pour l'analyse des laits de chèvre. Entre parenthèses : résultats d'études antérieures faites sur le lait de vache (Grappin et Jeunet, 1976 ; Grappin et Jeunet, 1978).

\begin{tabular}{|c|c|c|c|c|c|}
\hline \multirow{2}{*}{ Méthode } & \multicolumn{3}{|c|}{ Lait } & \multirow{2}{*}{$\begin{array}{l}\text { Sérum } \\
\text { T. PNC }\end{array}$} & \multirow{2}{*}{$\begin{array}{c}\text { Lait-sérum } \\
\text { T. PC }\end{array}$} \\
\hline & T. MG & T. MAT & T.P. & & \\
\hline Noir Amido & - & $\pm 1,4( \pm 0,8)$ & $\pm 0,8( \pm 0,7)$ & $\pm 0,37$ & $\pm 0,8$ \\
\hline MS 300 & $\pm 0,9( \pm 1,8)$ & $\pm 1,3( \pm 1,1)$ & $\pm 0,7( \pm 1,0)$ & $\pm 0,52$ & $\pm 0,5$ \\
\hline IRMA & $\pm 1,2( \pm 2,2)$ & $\pm 1,3( \pm 1,4)$ & $\pm 1,0( \pm 1,3)$ & - & - \\
\hline MTA & $\pm 1,0( \pm 1,3)$ & - & - & - & - \\
\hline \multicolumn{6}{|l|}{ T. MG } \\
\hline \multicolumn{6}{|c|}{ T. MAT : taux de matière azotée totale. } \\
\hline \multicolumn{6}{|c|}{ T.P. : : taux de protéines. } \\
\hline \multicolumn{6}{|c|}{ T. PNC : taux de protéines non coagulables. } \\
\hline \multicolumn{6}{|c|}{ T. PC : taux de protéines coagulables. } \\
\hline
\end{tabular}


Les résultats sont donnés pour les laits de chèvre de cette étude, dans laquelle nous avons mesuré les indices de réfraction de la matière grasse de vingt échantillons présentant les écarts les plus grands par rapport à la méthode Gerber, ainsi que pour les laits de vache (Grappin, Jeunet, 1978).

\section{II.3. Précision d'estimation}

Le tableau 4 donne pour chaque méthode la précision d'estimation du taux de matière grasse et des taux de matière azotée totale et de protéines du lait, ainsi que la précision d'estimation du taux de protéines non coagulables du sérum et du taux de protéines coagulables.

\section{II.4. Comparaison des calibrages lait de vache et lait de chèvre pour la détermination du taux de matière grasse et du taux de protéines}

Suivant la définition donnée en 1.3.2., nous avons porté dans le tableau 5 les équations de régression observées pour les laits de chèvre et de vache avec les différents appareils, la variable estimée y (taux de MG ou TP) étant la méthode de référence (§ I.2.1.). Pour le dosage des protéines du lait de vache, la méthode utilisée comme référence est la méthode au Noir Amido.

Ces différentes équations nous ont permis de comparer pour chaque appareil les valeurs estimées du taux de matière grasse ou du taux de protéines pour les laits de vache et les laits de chèvre, correspondant à une lecture donnée de l'appareil. En prenant comme exemple le dosage de la matière grasse avec l'IRMA (fig. 1) on constate qu'à une lecture donnée de l'appareil, soit 34,0 par exemple, correspondent des valeurs estimées du taux de matière grasse de 35,6 pour le lait de vache et 34,3 pour le lait de chèvre. Cela signifie qu'avec un taux de matière grasse plus faible de $1,3 \mathrm{~g} / \mathrm{kg}$, le lait de chèvre donne à l'IRMA, un résultat identique à celui du lait de vache. Le cœfficient d'absorption I.R. du lait de chèvre est donc, dans le cas présent, supérieur de 3,8 p. 100 à celui du lait de vache. Pour analyser des laits de chèvre à partir d'un appareil calibré pour doser des laits de vache, il faudrait donc dans cet exemple diminuer les lectures IRMA de $1,3 \mathrm{~g}$ au niveau de $34 \mathrm{~g}$.

Les différences portées dans le tableau 5 indiquent les corrections en grandeur et en signe qu'il faudrait, dans le cas particulier de cette étude, apporter aux appareils aux niveaux de $34 \mathrm{~g} / \mathrm{kg}$ pour la matière grasse et de $30 \mathrm{~g} / \mathrm{kg}$ pour les protéines pour analyser des laits de chèvre avec des appareils calibrés pour doser des laits de vache. 


\section{TABLEAU 5}

Comparaison des calibrages obtenus avec les différentes méthodes pour le dosage du taux de matière grasse et du taux de protéines des laits de vache et des laits de chèvre

Taux de matière grasse

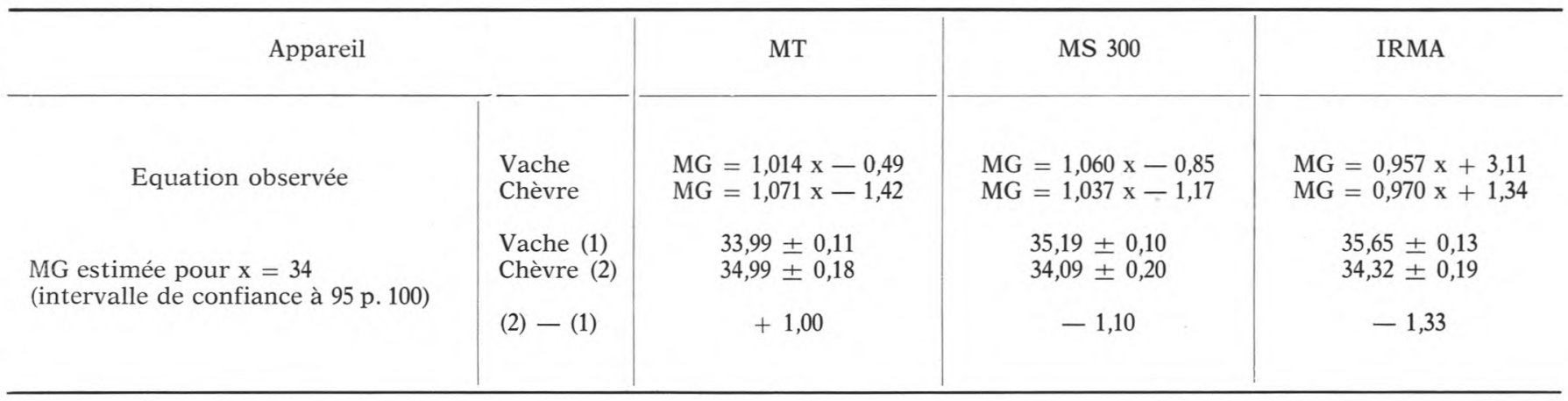

Taux de protéines

\begin{tabular}{|c|c|c|c|c|}
\hline Appareil & & Noir Amido & MS 300 & IRMA \\
\hline $\begin{array}{l}\text { TP estimé pour } \mathrm{x}=30 \\
\text { (intervalle de confiance à } 95 \text { p. 100) }\end{array}$ & $\begin{array}{l}\text { Vache } \\
\text { Chèvre } \\
\text { Vache (1) } \\
\text { Chèvre (2) } \\
(2)-(1)\end{array}$ & $\begin{array}{l}\mathrm{TP}=1,000 \mathrm{x} \\
\mathrm{TP}=1,007 \mathrm{x}-0,32 \\
30,00 \pm 0,07 \\
29,89 \pm 0,09 \\
-0,11\end{array}$ & $\begin{array}{c}\mathrm{TP}=1,021 \times-1,50 \\
\mathrm{TP}=1,052 \times-0,85 \\
29,13 \pm 0,08 \\
30,71 \pm 0,08 \\
+1,58\end{array}$ & $\begin{array}{c}\mathrm{TP}=1,147 \times-5,27 \\
\mathrm{TP}=1,105 \times-2,34 \\
29,14 \pm 0,09 \\
30,81 \pm 0,11 \\
+1,67\end{array}$ \\
\hline
\end{tabular}




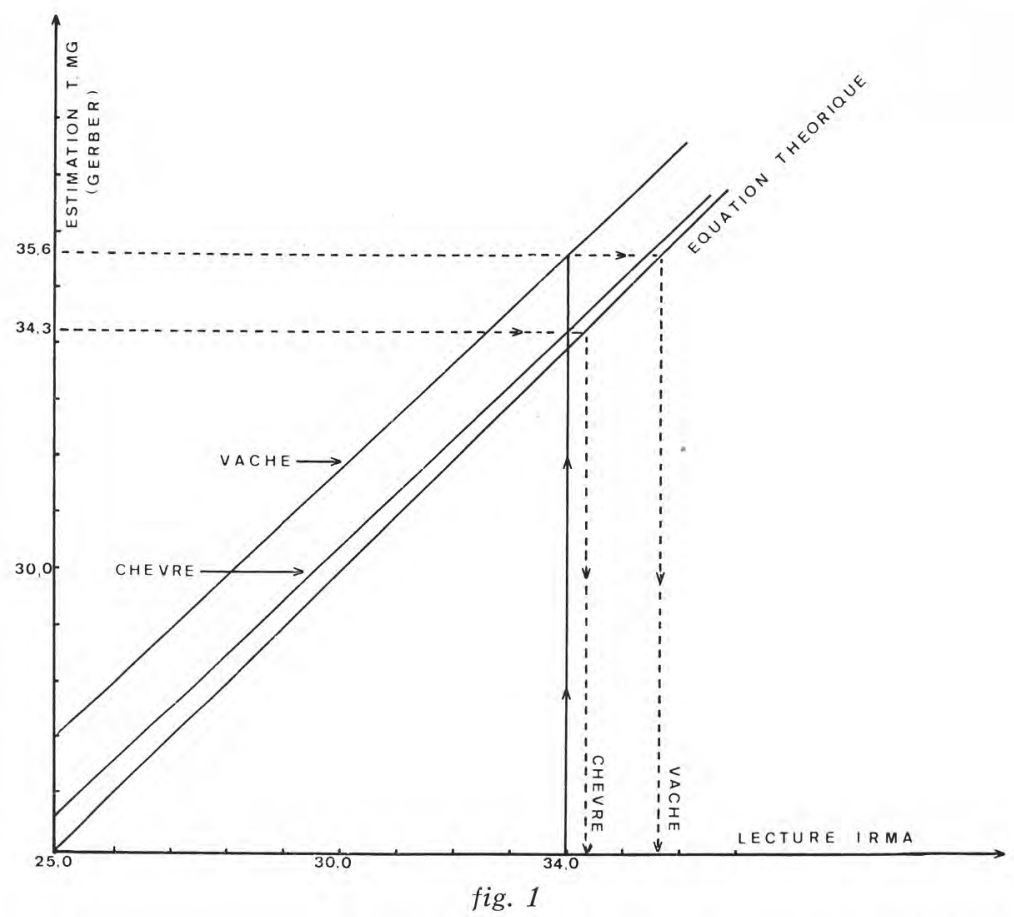

Comparaison des droites de calibrage observées avec l'appareil IRMA pour des laits de vache et des laits de chèvre

Vache : MG $=0,957$ IRMA $+3,11 \quad$ Chèvre : $M G=0,970$ IRMA $+1,34$

\section{DISCUSSION}

\section{III.1. Composition des laits}

Les résultats du tableau 1 montrent que, pour tous les constituants analysés, le lait de chèvre est en moyenne moins riche que le lait de vache. Ces résultats semblent caractéristiques des troupeaux laitiers caprins et bovins français mais sont difficilement comparables à ceux obtenus dans d'autres pays (Parkash et Jenness 1968). Soulignons que le lait de chèvre présente normalement un nombre élevé de cellules (Claveau, Grappin, Roguinsky, 1978) ; aussi peut-on admettre que malgré le nombre moyen relativement élevé de cellules trouvé dans notre étude, la plupart des échantillons provenaient vraisemblablement d'animaux ne présentant pas de mammite importante.

En ce qui concerne les fractions azotées (tab. 2), les laits de chèvre se caractérisent par une proportion de NPN dans la MAT 
environ deux fois plus élevée que celle du lait de vache (10 p. 100 contre 5 p. 100) et, par conséquent, par des proportions plus faibles de PS et surtout de caséines. Toutefois, si on ne considère que la fraction protéique du lait, le lait de chèvre présente, pour cette fraction, une proportion de caséine légèrement plus élevée que le lait de vache. Dans l'étude de 1972, cette différence, bien qu'étant relativement importante (1,5 p. 100) n'est cependant pas significative au seuil de probabilité de 5 p. 100.

\section{III.2. Dosage de la matière grasse}

Les valeurs de précision d'estimation portées au tableau 4 sont très bonnes comparativement à celles que nous avons obtenues précédemment avec les mêmes appareils dans le cas des laits individuels de vache (Grappin et Jeunet, 1978). En ce qui concerne les variations d'indice de réfraction de la matière grasse $\left(\mathrm{n}_{\mathrm{v}}\right)$ le tableau 3 montre, comme dans le cas des laits de vache, que ces variations semblent bien être à l'origine des écarts entre les appareils et la méthode Gerber. La faible variabilité des $n_{\text {s }}$ des échantillons de laits de chèvre analysés $\left(\sigma \mathrm{n}_{\mathrm{D}}=5 \cdot 10^{-4}\right)$ comparée à celle des laits individuels de vache analysés dans l'étude publiée en $1978\left(\sigma \mathrm{n}_{\mathrm{v}}=13.10^{-4}\right)$ explique la meilleure précision d'estimation des appareils dans le cas des laits de chèvre.

$\mathrm{Si}$ on considère maintenant les indices de réfraction moyens des échantillons de laits de vache $\left(n_{D}=1,45465\right)$ et de laits de chèvre $\left(n_{\mathrm{v}}=1,45280\right)$ analysés dans la présente étude, on constate que la différence de $18,5.10^{-4}$ de $n_{\text {v }}$ entre les deux types de lait peut expliquer les différences de calibrage. En effet, les résultats du tableau 3 (résultats vache 1978) nous permettent de calculer pour un lait à $34,0 \mathrm{~g} / \mathrm{kg}$ de $M G$ et une différence de $-18,5.10^{-4}$ de $n_{0}$ (lait de chèvre par rapport au lait de vache), des variations théoriques de $-0,94 \mathrm{~g} / \mathrm{kg}$ pour le MT, $+1,26 \mathrm{~g} / \mathrm{kg}$ pour le MS 300 et $+1,57 \mathrm{~g} / \mathrm{kg}$ pour I'IRMA.

Ces valeurs sont très proches de celles qui sont groupées dans le tableau 5. Les signes contraires du tableau 5 s'expliquent par le fait que ce sont des différences dans l'estimation du TB (donc des résultats Gerber) qui sont indiquées dans le tableau et non des différences de lecture sur les appareils.

Ces résultats montrent qu'il faut procéder à un calibrage particulier des appareils pour doser la matière grasse des laits de chèvre ainsi qu'à une vérification régulière de ce calibrage dans les mêmes conditions que celles qui sont déjà appliquées pour le dosage des laits de vache.

\section{III.3. Dosage des différentes fractions azotées}

La précision d'estimation des taux de MAT et de protéines des laits de chèvre qui a été obtenue avec les différents appareils (tab. 4) 
est voisine de celle qu'on a observée dans des conditions semblables avec le lait de vache (Grappin et Jeunet, 1976), avec toutefois une différence assez nette pour la détermination du taux de MAT par le Noir Amido ( $\pm 0,8$ pour le lait de vache, $\pm 1,4$ pour le lait de chèvre). Pour toutes les méthodes, on constate une précision d'estimation qui est meilleure pour le TP que pour le T.MAT. Ceci tient au fait que le NPN, non dosé par le Noir Amido et par les méthodes I.R. mais dosé par le Kjeldahl, est en proportion importante et relativement variable dans le lait de chèvre ( 9,3 p. 100 de la MAT, $\sigma=1,86$ ). Contrairement à ce qu'on observe généralement pour le lait de vache, la précision d'estimation de la méthode Noir Amido n'est pas meilleure que celle des appareils I.R.

Malgré une très bonne précision des dosages des protéines du sérum, la détermination du TPC par la méthode Noir Amido s'avère moins précise qu'avec le Milko-Scan ( $\pm 0,8$ contre $\pm 0,5 \mathrm{~g} / \mathrm{kg}$ ), et ceci bien que la précision d'estimation du T.PNC du sérum par le MS 300 ne soit pas très bonne $( \pm 0,52 \mathrm{~g} / \mathrm{kg}$ ).

Dans le cas du Noir Amido, les écarts assez importants observés dans l'estimation du TP du lait subsistent lorsqu'on fait la différence TP lait - T.PNC sérum pour la détermination du T.PC. En revanche, du fait d'une corrélation significative de $+0,60$ entre les écarts MS-Kjeldahl observés sur lait et les écarts MS-Kjeldahl observés sur sérum (fig. 3), les erreurs tendent à s'annuler, lorsqu'on fait la différence lait-sérum, permettant ainsi une détermination précise du T.PC par le MS.

\subsubsection{MÉthode NoIr AMIdo}

Dans l'estimation du TP par la méthode Noir Amido, on note que 67 p. 100 des différences par rapport au Kjeldahl sont expliquées par les variations du rapport T.PS/TP du lait (fig. 2). L'équation de la droite de régression montre qu'une augmentation de 1 p. 100 de ce rapport entraîne une surestimation des lectures Noir Amido de $0,55 \mathrm{p}$. 100 en valeur relative, soit $+0,16 \mathrm{~g} / \mathrm{kg}$ au niveau d'un lait dosant $30 \mathrm{~g}$ de protéines $/ \mathrm{kg}$. Cela signifie également que la capacité de fixation du colorant par les PS est environ 1,55 fois plus élevée que celle des caséines alors que ce rapport n'est que de 1,27 pour le lait de vache (Grappin et Jeunet, 1973). Cette différence associée à une assez grande variabilité du rapport T.PS/TP des laits de chèvre ( $\sigma=2,47$ dans l'étude de 1978) peuvent expliquer la précision relativement moins bonne de la méthode Noir Amido lorsqu'elle est appliquée aux laits de chèvre. Dans l'étude de 1972, la variabilité est plus faible $(\sigma=2,14)$ mais cependant significativement plus élevée que la variabilité du rapport T.PS/TP du lait de vache $(\sigma=1,93)$.

Le tableau 5 montre une faible différence de $0,11 \mathrm{~g} / \mathrm{kg}$ entre les deux droites de calibrage lait de chèvre et lait de vache indiquant que les protéines du lait de chèvre ont, dans le cas présent, une 


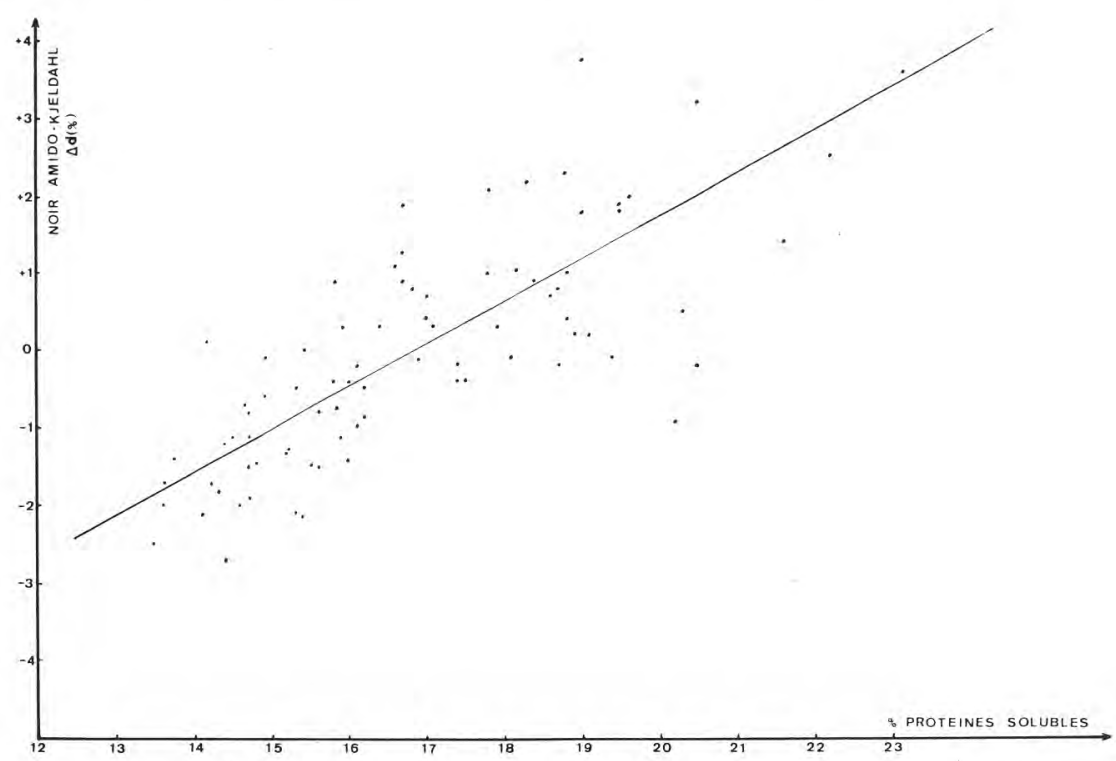

fig. 2

Dosage du taux de protéines des laits de chèvre par la méthode Noir Amido (NA). Relation entre les écarts Noir Amido-Kjeldahl $\Delta \mathrm{d}=$ (NA-Kjeldahl) 100 / Kjeldahl et la proportion de protéines solubles (p.100 PS $=$ T.PS/TP) des échantillons de lait.

$\Delta \mathrm{d}=0,55$ p. 100 PS $-9,33 \quad \mathrm{r}=0,82$.

capacité de fixation du colorant plus élevée de 0,4 p. 100 seulement par rapport à celle des protéines du lait de vache.

La méthode au Noir Amido étant utilisée en pratique comme méthode de référence secondaire pour le calibrage des appareils I.R., il serait important de bien définir les différences de calibrage pouvant exister entre les laits de chèvre et les laits de vache. Le calibrage de la méthode Noir Amido pour le dosage des laits de chèvre et des laits de vache dépend en fait, d'une part de la composition en caséines et en protéines solubles des laits et d'autre part de la capacité de fixation du colorant par les mêmes caséines et protéines solubles; ces deux paramètres pouvant avoir des valeurs différentes lorsqu'il s'agit de laits de vache ou de laits de chèvre. Si l'on possède des données précises sur ce sujet en ce qui concerne le lait de vache (Tarassuk et al., 1967), il n'existe en revanche que peu d'informations pour le lait de chèvre (Parkash et Jenness, 1968 ; Richardson et al., 1974).

\subsubsection{MÉTHOdes INFRA-ROUgE}

Malgré les différentes informations que nous possédons sur la composition des échantilllons de lait, il n'a pas été possible de relier 
les différences constatées entre les mesures I.R. et la méthode Kjeldahl à des caractéristiques particulières de composition des laits, et notamment à la teneur en lactose (Grappin et Jeunet, 1976).

Le cœfficient de corrélation faible mais significatif de $-0,23$ entre les écarts MS-Kjeldahl et le taux de lactose confirme l'influence de ce dernier dans la précision des dosages de protéines par les méthodes I.R. mais dans le cas présent cette influence est négligeable $\left(\mathrm{r}^{2}=0,05\right)$ compte tenu de la faible variabilité du taux de lactose.

Cependant, le fait que les écarts par rapport à la méthode Kjeldahl constatés sur les laits se retrouvent sur les dosages de sérum (fig. 3), laisse penser que ce sont les variations de composition des protéines solubles qui influenceraient en partie la précision des dosages I.R., le taux de lactose qui est l'autre élément important commun aux laits et aux sérums ayant une influence extrêmement réduite.

Quant à la différence de calibrage entre laits de vache et laits de chèvre, elle s'avère relativement importante, les protéines du lait de chèvre ayant un coefficient d'absorption I.R. environ 5 p. 100 plus faible que celui des protéines du lait de vache. Là également, nous ne possédons pas assez de renseignements sur la composition des laits pour expliquer cette différence.

Théoriquement, si l'on élimine l'influence des autres composants du lait, matière grasse et lactose, et l'influence du degré d'homogénéisation, la précision des dosages de protéines par les méthodes I.R. dépend de la seule variation du nombre de liaisons peptidiques par unité de poids, ou plus exactement par gramme d'azote si on se réfère à la méthode Kjeldahl. D’après la composition connue en acides aminés des principales fractions protéiques du lait de vache, on constate que le rapport nombre de résidus d'acides aminés / nombre d'atomes d'azote varie relativement peu suivant les fractions protéiques. On sait également que la composition en acides aminés des protéines du lait de chèvre est très voisine de celle des protéines correspondantes du lait de vache (Richardson et Creamer, 1974 et 1975). Par conséquent, pour expliquer les différences infra-rouge - Kjeldahl constatées soit entre échantillons de lait d'une même espèce, soit entre laits de vache et de chèvre, on peut émettre l'hypothèse que ce sont les proportions des différentes fractions azotées qui varient de façon importante entre animaux et entre espèces.

De récentes informations (Thompson, 1978) montrent par exemple qu'il existe effectivement une très grande variabilité des fractions caséiniques $\alpha_{\mathrm{s} 1}, \beta$ et $\chi$ entre vaches Holstein; de même, on sait (Richardson et al. 1974) que le lait de chèvre contient beaucoup moins de caséine $\alpha_{\mathrm{s}}$ que les caséines du lait de vache. En fait, seule une étude directe de la relation entre les mesures I.R. et Kjeldahl des protéines et la composition de ces protéines apportera une réponse satisfaisante à ce problème. 


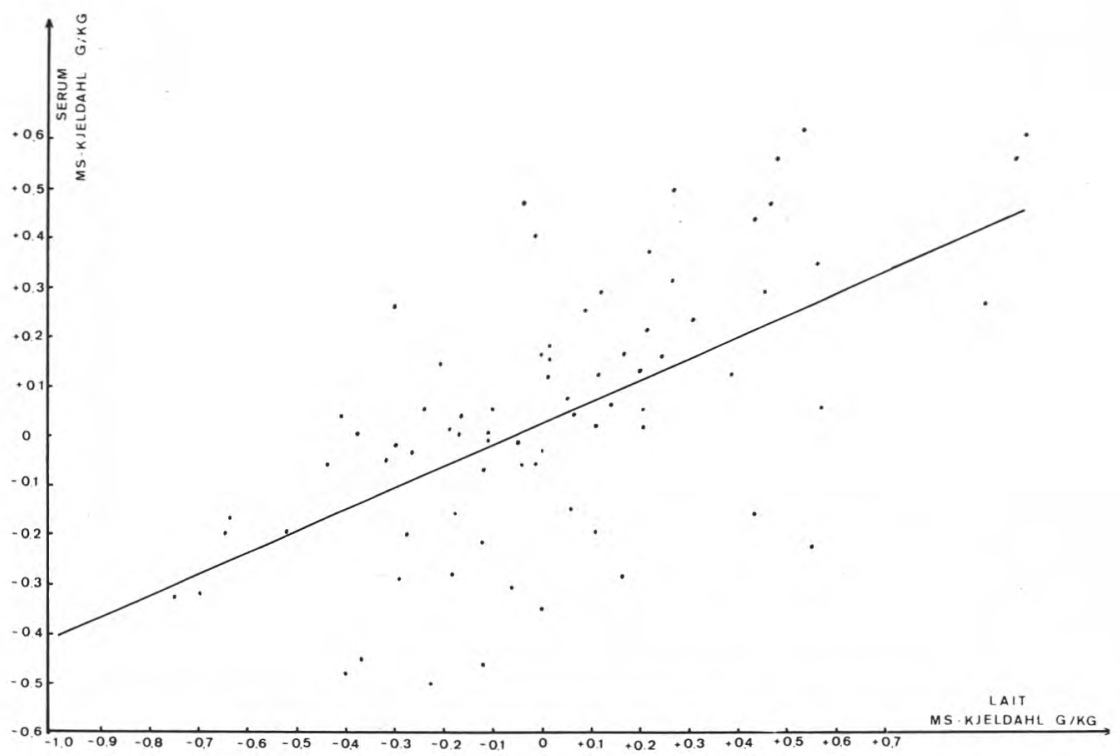

fig. 3

Dosage du taux de protéines des laits de chèvre et du taux de protéines non coagulables du sérum par les méthodes Kjeldahl et Milko-Scan 300

Relation entre les différences Milko-Scan - Kjeldahl (en g/kg) observées sur laits et sur lactosérums correspondants.

Equation de régression $\mathrm{y}=0,43 \mathrm{x}+0,02$.

Coefficient de corrélation $r=0,60$.

\section{CONCLUSION}

D'une manière générale, les précisions d'estimation du T.MG et du TP des laits de chèvre à l'aide des différentes méthodes de routine sont tout à fait comparables à celles qu'on observe avec le lait de vache. A cet égard, la méthode Noir Amido de même que les appareils Milko-Tester et I.R. peuvent donc être employés pour le dosage des échantillons de lait de chèvre destinés au contrôle laitier ou au paiement du lait suivant sa composition.

On peut souligner que la proportion élevée de NPN dans le lait de chèvre justifie plus encore que dans le cas du lait de vache, l'utilisation du TP et non du T.MAT pour définir la valeur protéique des laits. 
Les différences constatées par rapport aux méthodes de référence s'expliquent de manière semblable pour les laits de vache et les laits de chèvre : variation de l'indice de réfraction de la matière grasse pour le dosage de matière grasse avec les différents appareils et rapport T.PS/TP pour le dosage des protéines par la méthode Noir Amido.

L'utilisation des méthodes de routine pour le dosage de la matière grasse et des protéines du lait de chèvre nécessite des calibrages différents de ceux utilisés dans le cas des laits de vache. Il faut noter que les différences observées dans cette étude (tab. 5) ne doivent pas être considérées comme définitives, car elles peuvent varier avec la saison et la région. Les raisons de ces différences ne sont pas encore clairement établies, en particulier dans le cas du dosage des protéines par les méthodes infra-rouge. Une étude est en cours sur cette question.

\section{Rés u mé}

Quatre-vingt-un échantillons individuels de lait de chèvre ont été analysés en matière grasse par les méthodes Gerber (référence), Milko-Tester (MT), Milko-Scan 300 (MS) et IRMA, et en protéines (N total - NPN) x 6,39 par les méthodes Kjeldahl (référence), Noir Amido (NA), MS et IRMA.

La précision d'estimation $\left( \pm 1,96 \sigma_{y, x}\right)$ du taux de matière grasse et du taux de protéines exprimées en $\mathrm{g} / \mathrm{kg}$ est la suivante : $\mathrm{MT}=$ $\pm 1,0$; MS $= \pm 0,9$; IRMA $= \pm 1,2$ pour le taux de matière grasse et $\mathrm{NA}= \pm 0,8 ; \mathrm{MS}= \pm 0,7$; IRMA $= \pm 1,0$ pour le taux de proteines.

Des mesures du taux de matière azotée totale ( $\mathrm{N}$ total $\mathrm{x} 6,39$ ), du taux de protéines coagulables par la présure et du taux de protéines du sérum ont également été effectuées.

Les différences par rapport à la méthode Gerber, pour le dosage des matières grasses, sont expliquées par les variations d'indice de réfraction de la graisse. Les différences NA-Kjeldahl de la mesure du taux de protéines viennent principalement des variations du rapport protéines solubles / caséines.

Des différences importantes de calibrage sont observées avec les différentes méthodes entre les laits de chèvre et les laits de vache. Elles s'expliquent généralement par des différences de composition. Pour l'ensemble des méthodes étudiées, il est recommandé d'utiliser des calibrages particuliers pour le dosage des matières grasses et des protéines des laits de vache et des laits de chèvre. 


\section{S u m m a r y}

The composition of 81 individual goats milk samples was determined by Gerber (as a reference), Milko-Tester (MT), Milko-Scan 300 (MS) and Infra-red Milk Analyser (IRMA) for fat content and by Kjeldahl (as a reference), Amido Black (A.B) MS and IRMA for true protein content (total N-NPN) $\times$ 6.39. Determinations of the protein content (total $\mathrm{N} \times 6.39$ ) and rennet whey proteins were also carried out. The accuracy of estimate $\left( \pm 1.96 \sigma_{y, x}\right)$ for fat and true protein contents, expressed as percent, is as follows: $\mathrm{MT}= \pm 0.10$; $\mathrm{MS}= \pm 0.09 ; \mathrm{IRMA}= \pm 0.12$ for fat and $\mathrm{AB}= \pm 0.08 ; \mathrm{MS}= \pm 0.07$; IRMA $= \pm 0.10$ for protein.

Differences between Gerber and the other tests for fat can be explained by the variability of the butterfat refractive index. Differences in true protein between $\mathrm{Kjeldahl}$ and $\mathrm{AB}$ are mainly due to the variability of the whey protein / casein ratio of the milk samples.

Significant differences were observed between calibrations curves for cows and goats milk with the various test procedures. For all the methods involved specific calibrations are recommended for cows and goats milk both for fat and for protein.

Reçu pour publication en mars 1979.

\section{Références bibliographiques}

RoWLAND (S. J.) (1938), - The determination of nitrogen distribution in milk. J. Dairy Res., 9, 42-46.

GRAPPIN (R.) (1976). - Guide pour l'évaluation des méthodes d'analyses de routine. Le Lait, 56, 608-621.

Claveau (R.), Grappin (R.), Roguinsky (M.) (1978). - Etude préliminaire des méthodes de numération des cellules du lait appliquées au lait de chèvre. $20^{\text {e }}$ Congrès Intern, de Laiterie, Paris, 353.

GRAPPIN (R.), JeUnet (R.) (1978). - Comparaison des méthodes actuelles de dosage de la matière grasse du lait. $20^{\circ}$ Congrès Intern. de Laiterie, Paris, 205-206.

GrapPin (R.), Jeunet (R.) (1976). - Calibrage des méthodes de dosage de routine des protéines du lait. Rev. Lait. Franç., 347, 663-667.

Grappin (R.), JeunET (R.) (1973). - Influence de la composition de la matière azotée du lait sur la précision des dosages de matière azotée par la méthode au Noir Amido. Conséquences. Réunion Cercle Intern. des Chercheurs Laitiers, Poligny, 15-17 mai 1973.

Grappin (R.), Jeunet (R.) (1976). - Essais de l'appareil Milko-Scan 300 utilisé pour le dosage en série de la matière grasse et des protéines du lait. Le Lait, $56,498-520$.

PARKASH (S.), Jenness (R.) (1968). - The composition and characteristics of goat's milk. A review: Dairy Sc. Abstr., 30, 67-87. 
Richardson (B. C.), Creamer (L. K.), Munford (R. E.) (1974). - The composition and properties of caprine caseins. XIX inter. Dairy Cong. $1 \mathrm{E}, 268$.

Richardson (B. C.), Creamer (L. K.) (1974). - Comparative micelle structure. The isolation and chemical charaterization of caprine $\beta_{1}$ and $\beta_{2}$ casein. Bioch. Bioph. Act., 365, 133-137.

Richardson (B. C.), CREAMER (L. K.) (1975). - Comparative micelle structure. IV. The similarity between $\alpha_{\mathrm{s}}$ casein and bovine $\alpha_{\mathbb{S} 3}$ casein. Bioch. Bioph. Act., 393, 37-47.

Tarassuk (N. P.), Abe (N.), Moats (W. A.) (1967). - The dye-binding of milk proteins. Tech. Bull. U.S.D.A., 1369, 1-44.

Thompson (M. P.) (1978). - Determination of casein composition by gel densitometry. Midwest milk and food protein seminar, 29-30 Sept. 1978. University of Minn., St Paul. 